\title{
Oceans cool off in hottest years
}

Ocean measurements suggest the world's seas have cooled substantially during some of the warmest years in recent history. If real, the dip is likely to reflect a short-term fluctuation in an ocean that is warming overall, say climate scientists.

The years 2003 and 2005 saw the highest global average surface temperatures in more than a century. An upcoming paper in Geophysical Research Letters reports that during this period, the upper 750 metres of the oceans lost around one-fifth of the heat accumulated over the past 50 years.

But don't read too much into that, oceanographers warn. "Cooling on a short-term scale doesn't challenge the long-term warming trend," says Sydney Levitus, director of the World Data Center for Oceanography in Silver Spring, Maryland. "What it does tell us is that we still don't sufficiently understand how the global dimate system works."

Oceans cover two-thirds of Earth's surface and have far more heat-storing capacity than air or land. Overall, they have warmed in recent decades. Between 1955 and 1998, for example, all of the world's oceans, down to a depth of 3,000 metres, warmed by $0.037^{\circ} \mathrm{C}$ (S. Levitus, J. Antonov and T. Boyer Geophys. Res. Lett. 32, L02604; 2005).
But that warming has not proceeded steadily. The trend has reversed for a few years at least once during the past half century. In fact, a drop in temperature that occurred between 1980 and 1983 was almost twice as pronounced as the current cooling.

But only for this latest cooling episode have oceanographers obtained good enough global data to provide a convincing argument that the effect is real. The report relies in part on data gathered by ARGO, a global array of 2,500 floats. The ARGO floats descend to various depths to probe ocean temperature and salinity, and surface occasionally to transmit

"We may be seeing different things just because we are looking harder." their data via satellites.

When ARGO data were removed from the analysis, the cooling was significantly less pronounced, and the error bars became larger, say the authors of the report, led by John Lyman of the National Oceanic and Atmospheric Administration in Seattle, Washington. Some researchers suspect that ARGO has provided the ability to detect short-term temperature fluctuations. ${ }^{\alpha}$ We may be seeing different things just because we are looking harder," says Brian King, a physical oceanographer at the Southampton Oceanography Centre, UK.
Better observation systems are showing the oceans are more changeable than some people thought, says Gavin Schmidt, a climate modeller at the NASA Goddard Institute for Space Studies in New York City. A short-term cooling blip can't say much about the climate system in general. But the latest study suggests, he says, that coupled ocean-climate models fail to adequately capture intermittent fluctuations in ocean temperature.

In theory, the recent cooling should have led to a 2-millimetre drop in sea level due to the thermal contraction of water, which becomes denser at lower temperatures. But satellites have measured a steady rise in sea level between 1993 and 2005 - years covered by the study. For the observations to be reconciled, melting ice sheets in Antarctica and Greenland must be contributing far more to sea-level rise than previously suspected, says Schmidt.

Of course, much of the observed ocean-temperature decrease could be an artefact of sampling, says Tim Barnett, a climate researcher at the Scripps Institution of Oceanography in San Diego, California. For example, if warmer water has simply redistributed to areas where fewer ARGO floats exist, the net cooling 


\section{Asteroid fly-by eludes study}

On 3 July, an asteroid zipped past Earth at a distance of some 400,000 kilometres slightly farther away than the Moon. In theory, something that close ought to be easy to study. But astronomers have struggled tomap the size and shape of the space rock - and now say they know why they found itso difficult.

The rock, dubbed 2004 XP14, is one of more than $\mathbf{8 0 0}$ 'near-Earth asteroids' that have been identified in orbits that come perilously close to our planet. This particular rock is unlikely to hit us, but astronomershoped their observations would help establish how diverse such asteroids are and so better quantify the threat they pose.

But the data obtained by the team proved surprisingly hard to analyse. "The asteroid rotatesslowly, so its appearance in the images, due to rotation, hardly changed atall," says Lance Benner, an astronomer at NASA's Jet Propulsion Laboratory in Pasadena, California, who led the effort to image the asteroid.

That's unusual. "Most near-Earth asteroids are very fast rotators," says Vishnu Reddy, a graduate student at the University of North Dakota who alsoobserved the object.

Benner and his colleagues imaged 2004 XP14 using a 70-metre radio antenna at the Goldstone Complex in California. At260metres across, the asteroid was a lot smaller than earlier predictions of up to 880 metres. This, together with its rotation rate of roughly one turnevery $\mathbf{5 0 0}$ hours, meant that the images the team received barely changed during recording sessions of up to 2 hours. As a result, the researchers couldnot build the detailed picture of the rock that they wanted.

Earth may be safe from 2004 XP14, but there are plenty more asteroids out there that might collide with us. Having identified threequarters of the candidates 1 kilometre or more in diameter, NASA plans to widen its search to include objects as small as $\mathbf{1 5 0}$ metres across. And in July, the International Astronomical Union formed a committee to keep it up to date about aster oids that may pose a serious threat to our planet.

Heidi Ledford

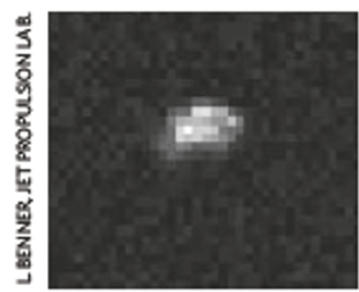

Blip on the horizon: radar imaging of the asteroid 2004 XP14 proved to be more difficult than expected. well be larger than we thought," says Levitus. "This is exciting news, but food for global-warming sceptics it is not.

Quirin Schiermeier 